\title{
Erratum to: Frontier Computing
}

Jason C. Hung, Neil Y. Yen, and Lin Hui

\section{Erratum to: \\ J. C. Hung et al. (Eds.): Frontier Computing, LNEE 464, https://doi.org/10.1007/978-981-10-7398-4}

The original version of the book was inadvertently published without the affiliation "School of Computer Science and Engineering, University of Aizu, Aizu-Wakamatsu, Japan" of second editor "Neil Y. Yen" in frontmatter, which has to be now included. The erratum book has been updated with the change. 